\title{
Transient Ischaemic Attack Rarely Precedes Stroke in a Cohort with Low Proportions of Large Artery Atherosclerosis: A Population-Based Study
}

\author{
Blake F. Giarola a, b James Leyden ${ }^{c}$ Sally Castle ${ }^{c} \quad$ Jim Jannes ${ }^{d, ~ e ~}$ \\ Craig Anderson $^{b, f}$ Jonathan Newburye, $g$ Timothy Kleinig ${ }^{d, e}$
}

${ }^{a}$ Neurology Department, St Vincent's Hospital, Sydney, NSW, Australia; ${ }^{b}$ University of

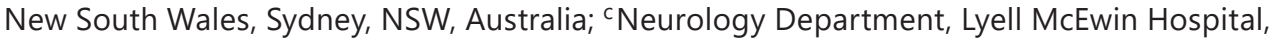
Adelaide, SA, Australia; 'Stroke and Neurology Department, Royal Adelaide Hospital, Adelaide, SA, Australia; ' University of Adelaide, Adelaide, SA, Australia; ${ }^{\mathrm{f}}$ Neurology Department, Royal Prince Alfred Hospital, Sydney, NSW, Australia; ${ }^{9}$ Rural Clinical School, University of Adelaide, Adelaide, SA, Australia

\section{Keywords}

Large artery atherosclerosis - Transient ischaemic attack · Ischaemic stroke - Adelaide Stroke Incidence Study

\begin{abstract}
Background: Ischaemic stroke is reportedly preceded by transient ischaemic attack (TIA) in $15-30 \%$ of all cases. The risk of stroke following TIA is highest in the presence of unstable atherosclerotic plaques in large arteries. The recent population-based Adelaide Stroke Incidence Study describes a population with a low proportion (16\%) of stroke attributable to large artery atherosclerosis (LAA). We hypothesized that this population-based ischaemic stroke cohort would have a lower rate of preceding TIA than previously reported. Methods: This paper is a prospective ascertainment of all suspected TIAs and strokes in a 12-month period from 2009 to 2010. Ischaemic stroke pathogenesis was classified by the TOAST criteria. Details
\end{abstract}

Jim Jannes is Associate Professor of Neurology, Head of Neurology Department at Royal Adelaide Hospital. Craig Anderson is Executive Director, Professor of Neurology and Epidemiology, Faculty of Medicine, University of New South Wales Sydney. Jonathan Newbury is Adjunct Professor of Rural Health, Adelaide Rural Clinical School, University of Adelaide. Timothy Kleinig is Associate Professor of Neurology, Head of Stroke Department at Royal Adelaide Hospital. 
of preceding TIA events were scrutinised. Results: In this 12-month period, 318 stroke events in 301 individuals were recorded. Of the total 258 ischaemic strokes, 16\% (95\% confidence interval [Cl] 12-22) were from LAA. Of 258 ischaemic stroke patients, only 11 (4\%; $95 \% \mathrm{Cl} 2-7$ ) reported symptoms in the preceding 90 days consistent with TIA. Nine (82\%) sought medical attention. The median $A B C D^{2}$ score in this group was 4.5 (IQR: 3-7), and the median time of event prior to stroke was 20 days (IQR: 4-32). Conclusion: In our population-based cohort, rates of TIA preceding ischaemic stroke were much lower than previously reported, probably reflective of effective secondary prevention (active TIA clinics) and primary prevention (limiting LAA prevalence). In our population, further enhancements in TIA care will be of limited yield.

\section{Introduction}

Transient ischaemic attack (TIA) is a harbinger of stroke with $15-30 \%$ of all strokes being preceded by TIA $[1,2]$. The risk of subsequent stroke is greatest immediately following a TIA. Natural history studies suggest a 90 -day stroke-risk of $10 \%$, of which half occur in the first 2 days [3].

The risk of recurrent stroke is directly related to the underlying aetiology [4]. Of all TIA aetiologies, large artery atherosclerosis (LAA) has been established as the highest risk for subsequent progression to stroke [5, 6]. CT and MRI angiography have significantly improved the risk stratification by identifying high-risk stenosis, which is present in almost $50 \%$ of post TIA imaging $[7,8]$.

In the recent Adelaide Stroke Incidence Study (ASCEND), in a population receiving high rates of primary or secondary preventative medication, the rates of LAA as a cause of ischaemic stroke were low (16\%) [9]. This population was also served by an active rapid-access TIA clinic. It was hypothesized that the rates of TIA preceding a stroke in this population would also be low.

\section{Methods}

\section{Definition}

As per guidelines for an "ideal" population-based stroke study $[9,10]$, stroke was defined as per the World Health Organisation (WHO) criteria. TIA was defined as "stroke symptoms and signs that resolve within $24 \mathrm{~h} . "$ Ischaemic stroke pathogenesis was classified by the Trial of ORG 10172 in Acute Stroke Treatment (TOAST) criteria [11].

\section{Ascertainment}

The ASCEND study followed criteria of an ideal stroke ascertainment model (see online Appendix 1; for all online suppl. material, see www.karger.com/doi/10.1159/000491936). It captured all potential strokes in a population of 148,000 between July 2009 and July 2010. This included all "TIAs" to determine whether symptom duration was truly $<24 \mathrm{~h}$. Patients with "tissue-based" strokes (previously defined as TIAs) were recorded during ascertainment but excluded from stroke incidence numbers to improve the comparability with previous studies. All patients with potential stroke or TIA were screened in this 12-month period with the use of standardized data collection forms. $\mathrm{ABCD}^{2}$ scores were obtained for TIAs prospectively, retrospectively from medical records where preceding TIA occurred outside the 1-year study period ( 1 case), and from interviews when the patient did not present to medical attention (1 case). 


\section{Case Analysis}

All patients with transient symptoms were reviewed to determine whether symptoms lasted longer than $24 \mathrm{~h}$. From each stroke patient, extensive medical history was obtained, including the dates of preceding TIAs. Each potential stroke was assessed against the inclusion criteria by 2 neurologists, with reference to a third neurologist in the case of disagreement. Cases were included in which a diagnosis of probable stroke could be made by consensus.

\section{Data Analyses}

The clinical details and results were recorded into a purpose-built database. The incidence was calculated per 100,000 per year with 95\% confidence intervals (CIs) via Poisson distribution.

\section{Results}

The study ascertained 318 strokes in 301 patients. Of the 318 strokes, excluding intracerebral haemorrhage, subarachnoid haemorrhage, and undetermined strokes, 258 strokes were ischaemic. Of 258 ischaemic strokes, 31 patients (12\%; 95\% CI 9-17) had a lifetime TIA. Three patients (1\%) reported a history of more than 1 TIA at any point in their life.

Only 11 patients ( $4 \%$; 95\% CI 2-7) had an acute ( $<90$ day) history of TIA. Nine ( $82 \%)$ had sought attention in the emergency department, and $3(27 \%)$ were subsequently referred to a TIA clinic. One patient sought follow-up with a private neurologist, and 1 patient did not seek medical attention until his/her stroke event. Nine (82\%) TIAs were captured prospectively; 2 were retrospectively reviewed ( 1 verified from private medical records, and 1 via retrospective history alone). Six patients (55\%) received CT at TIA diagnosis, 1 of whom showed evidence of acute infarction. Two received an MRI, of whom 1 had evidence of acute infarction (with normal CT). Five received no neuroimaging.

Of the 258 patients with ischaemic stroke, $42 \%$ were of cardioembolic aetiology, $25 \%$ were undetermined, $16 \%$ were LAA, $11 \%$ were small-vessel disease, and $6 \%$ were of other origins. Of the patients with preceding TIA within 90 days, 55\% were of cardioembolic aetiology, $27 \%$ were undetermined, and $18 \%$ were small-vessel disease. The median time of the event prior to stroke was 20 days (IQR: 4-32), and the median $\mathrm{ABCD}^{2}$ score was 4 (range 2-7).

Of all patients with ischaemic stroke, $159(62 \%)$ were previously actively treated for hypertension, 104 patients (40\%) were receiving lipid-lowering medications, and 130 (50\%) had a history of smoking. A total of $156(60 \%)$ of the 258 patients with ischaemic stroke were on at least 1 antiplatelet. Of the 11 patients with recent TIA, on admission to hospital following stroke, 9 (82\%) of the patients were on at least 1 antihypertensive, $6(55 \%)$ were on lipidlowering therapy, and 6 (55\%) had a history of smoking, 2 current. Nine patients (82\%) were receiving antithrombotic therapy at the time of stroke (online suppl. Table).

\section{Discussion}

It has been previously reported that the $\mathrm{ABCD}^{2}$ score performs poorly in Australia with low absolute 90 -day stroke rates following TIA [12]. From this it could be hypothesised that few patients in Australia with stroke would have an acute pre-stroke TIA. This was confirmed in the ASCEND study. Stroke in our cohort was preceded by TIA within the preceding 90 days in only $4 \%$ of the patients with ischaemic stroke, substantially lower than previous estimates.

The potential reasons for this finding are complex. Australia has universal health care, with relatively high rates of statin-prescription, anti-hypertensive treatment and low smoking 
rates. Low rates of LAA in ASCEND were believed to reflect these factors [13, 14]. Of patients with prior TIA, three-quarters were on lipid-lowering therapy, and most received antithrombotic therapy. Only 3 of the 11 patients were current smokers.

Intensive therapy following the diagnosis of minor stroke or TIA reduces the stroke risk by $80 \%$ [2]. The ASCEND population was served by active stroke units and rapid-access TIA clinics, in which many of the patients with transient symptoms were seen (see online suppl. Appendix 2). As rapid appropriate treatment of TIA lowers ischaemia recurrence, our study most likely reflects effective secondary (as well as primary) prevention.

Our study had several limitations. As all possible or probable TIAs were prospectively ascertained for 12 months (simultaneously to strokes), it is possible that we may not have identified all TIAs in the 90 days prior to study commencement. In addition, the diagnostic panel did not independently review all transient neurological events to assign a definite TIA diagnosis, nor did they routinely speak with all patients' relatives or treating medical practitioner(s) to help ascertain symptoms that may have potentially represented TIA.

\section{Summary}

In our population-based study, stroke was rarely preceded by acute or subacute TIA. While this is discordant to previous reports, it is not necessarily contradictory or counterintuitive. It probably reflects the effective primary and secondary prevention measures in our cohort and was in part mediated by low proportions of LAA-related stroke. Further refinements in the treatment of patients with TIA in our population would be unlikely to lead to substantial reductions in the burden of stroke.

\section{Acknowledgments}

Julie-Anne Greutner and the SEARCH investigators.

The Stroke Epidemiology in an Australian Rural Cohort study was funded by the National Health and Medical Research Council as the "Incidence and outcome of stroke in rural Australia," project grant 565402, 2009-2011.

\section{Disclosure Statement}

The authors declare no conflicts of interest.

\section{References}

1 Easton JD, Saver JL, Albers GW, Alberts MJ, Chaturvedi S, Feldmann E, et al: Definition and evaluation of transient ischemic attack: a scientific statement for healthcare professionals from the American Heart Association/American Stroke Association Stroke Council; Council on Cardiovascular Surgery and Anesthesia; Council on Cardiovascular Radiology and Intervention; Council on Cardiovascular Nursing; and the Interdisciplinary Council on Peripheral Vascular Disease. The American Academy of Neurology affirms the value of this statement as an educational tool for neurologists. Stroke 2009;40:2276-2293.

2 Rothwell PM, Warlow CP: Timing of TIAs preceding stroke: time window for prevention is very short. Neurology 2005;64:817-820.

3 Rothwell PM, Giles MF, Chandratheva A, Marquardt L, Geraghty O, Redgrave JN, et al: Effect of urgent treatment of transient ischaemic attack and minor stroke on early recurrent stroke (EXPRESS study): a prospective population-based sequential comparison. Lancet 2007;370:1432-1442. 
4 Purroy F, Montaner J, Molina CA, Delgado P, Ribo M, Alvarez-Sabin J: Patterns and predictors of early risk of recurrence after transient ischemic attack with respect to etiologic subtypes. Stroke 2007;38:3225-3229.

5 Lovett JK, Coull AJ, and Rothwell PM: Early risk of recurrence by subtype of ischemic stroke in populationbased incidence studies. Neurology 2004;62:569-573.

6 Chimowitz MI, Lynn MJ, Howlett-Smith H, Stern BJ, Hertzberg VS, Frankel MR, et al: Comparison of warfarin and aspirin for symptomatic intracranial arterial stenosis. N Engl J Med 2005;352:1305-1316.

7 Giles MF, Albers GW, Amarenco P, Arsava EM, Asimos AW, Ay H, et al: Early stroke risk and ABCD2 score performance in tissue- vs time-defined TIA: a multicenter study. Neurology 2011;77:1222-1228.

8 Prabhakaran S, Chong JY, Sacco RL: Impact of abnormal diffusion-weighted imaging results on short-term outcome following transient ischemic attack. Arch Neurol 2007;64:1105-1109.

9 Leyden JM, Kleinig TJ, Newbury J, Castle S, Cranefield J, Anderson CS, et al: Adelaide Stroke Incidence Study: declining stroke rates but many preventable cardioembolic strokes. Stroke 2013;44:1226-1231.

10 Sudlow C and Warlow C: Getting the priorities right for stroke care. BMJ 2009;338:2083.

11 Adams HP, Bendixen BH, Kappelle LJ, Biller J, Love BB, Gordon DL, et al: Classification of subtype of acute ischemic stroke. Definitions for use in a multicenter clinical trial. TOAST. Trial of Org 10172 in Acute Stroke Treatment. Stroke 1993;24:35-41.

12 Sanders LM, Srikanth VK, Psihogios H, Wong KK, Ramsay D, Phan TG: Clinical predictive value of the ABCD2 score for early risk of stroke in patients who have had transient ischaemic attack and who present to an Australian tertiary hospital. Med J Aust 2011;194:135-138.

13 Ovbiagele B, Goldstein LB, Amarenco P, Messig M, Sillesen H, Callahan A, et al: Prediction of major vascular events after stroke: the stroke prevention by aggressive reduction in cholesterol levels trial. J Stroke Cerebrovasc Dis 2014;23:778-784.

14 Arima H, Anderson C, Omae T, Woodward M, MacMahon S, Mancia G, et al: Degree of blood pressure reduction and recurrent stroke: the PROGRESS trial. J Neurol Neurosurg Psychiatry 2014;85:1284-1285. 